Chem. Pharm. Bull.

36( 9 )3468-3473(1988)

\title{
High-Performance Liquid Chromatographic Analysis of Acidic Saponins of Ginseng and Related Plants
}

\author{
Hiroyuki Yamaguchi, ${ }^{*, a}$ Ryoji Kasai, ${ }^{b}$ Hiromichi Matsuura, ${ }^{a}$ \\ OSAMU TANAKA ${ }^{b}$ and TORU FUWA ${ }^{a}$
}

\author{
Central Research Laboratories, Wakunaga Pharmaceutical Co., Ltd., ${ }^{a}$ Shimokotachi 1624, \\ Koda-cho, Takata-gun, Hiroshima 729-64, Japan and Institute of Pharmaceutical \\ Sciences, Hiroshima University School of Medicine, ${ }^{b}$ Kasumi, \\ Minami-ku, Hiroshima 734, Japan
}

(Received February 26, 1988)

\begin{abstract}
High-performance liquid chromatography (HPLC) of the glucuronide saponin (ginsenoside$\mathrm{Ro}$ ) and malonyl-saponins (malonyl-ginsenosides- $R \mathrm{~b}_{1},-\mathrm{Rb_{2 }},-\mathrm{Rc}$ and $-\mathrm{Rd}$ ) in ginseng root was investigated. Separation of these acidic saponins as well as the major neutral saponins could be achieved by HPLC either on an octadecyl silica column or on an amino column by using aqueous acetonitrile containing $\mathrm{KH}_{2} \mathrm{PO}_{4}$ (the former column) or $\mathrm{H}_{3} \mathrm{PO}_{4}$ (the latter column) as the mobile phase. By means of this procedure, the contents of acidic and neutral saponins of Panax ginseng, $P$. quinquefolium (American ginseng), $P$. japonicus collected in South Kyushu, P. notoginseng (Sanchiginseng) and Panax species collected at central Nepal were determined and compared.
\end{abstract}

Keywords_— _ ginseng; saponin; glucuronide; malonyl-ginsenoside; ginsenoside; HPLC; Panax species; Araliaceae; American ginseng; Sanchi-ginseng

A number of neutral dammarane-saponins have been isolated from ginseng, the root of Panax ginseng C.A. MEYER, and the pharmacological studies on ginseng have centered on the major neutral saponins, ginsenosides- $R b_{1}(1),-R b_{2}(2),-\operatorname{Rc}(3),-\operatorname{Rd}(4),-\operatorname{Re}(5),-\operatorname{Rf}(6)$ and $-\mathrm{Rg}_{1}(7)$, which are characteristic of Panax spp. ${ }^{1)}$

Recently, Kitagawa et al. ${ }^{2)}$ reported the isolation of acidic malonates of the dammarane saponins, malonyl-ginsenosides- $\mathrm{Rb}_{1}(\mathbf{8}),-\mathrm{Rb}_{2}(\mathbf{9})$ and $-\mathrm{Rd}(\mathbf{1 0})$ from ginseng in high yields. Malonyl-ginsenoside-Rd (11) was also isolated in a relatively low yield. Because these malonyl-saponins are readily demalonylated by heating to give the parent neutral ginsenosides, 8-11 are present in neither so-called red ginseng (steamed ginseng) nor extracts with boiling solvents. Together with these acidic malonyl-saponins, another acidic saponin, ginsenoside- $\left.\mathrm{Ro}^{3}\right)(\mathbf{1 2})$, which is a saponin of oleanolic acid (13) having a glucuronide unit in the sugar moiety, was also isolated from this crude drug. This saponin is identical with chikusetsusaponin V which was first isolated from rhizomes of Panax japonicus C.A. MEYER by Shoji et al. ${ }^{4)}$

High-performance liquid chromatography (HPLC) of the neutral saponins of ginseng has been studied extensively. Recently, we have reported that borate ion-exchange HPLC, ${ }^{5)}$ as well as normal phase HPLC on a column of new hard spherical hydroxyapatite, ${ }^{6)}$ is useful for the separative analysis of these neutral saponins. However, no report on analysis of the acidic saponins of ginseng has appeared in the literature. The present paper deals with an HPLC study of the acidic saponins and describes an application to the quantitative analysis of saponins of some Panax spp.

\section{Analysis of Acidic and Neutral Saponins}

For the HPLC separation of ginseng neutral saponins on an octadecyl silica (ODS) 
column, a mixture of acetonitrile and water has generally been used as the mobile phase. However, with this neutral solvent system, analysis of the acidic saponins did not afford a satisfactory result. The HPLC separation of major acidic and neutral saponins was conducted by addition of $\mathrm{KH}_{2} \mathrm{PO}_{4}$ to the mobile phase. The correlation of concentration of $\mathrm{KH}_{2} \mathrm{PO}_{4}$ with capacity ratio $\left(k^{\prime}\right)$ of the saponins was explored (Fig. 1), indicating a decrease of $k^{\prime}$ with more than $50 \mathrm{~mm} \mathrm{KH}_{2} \mathrm{PO}_{4}$. Excellent separation of the major saponins, except 5 and 7, was obtained with the mobile phase containing $50 \mathrm{~mm} \mathrm{KH}_{2} \mathrm{PO}_{4}$ in $31 \%$ aqueous acetonitrile (condition A, Fig. 2). As for quantitative analysis by HPLC under condition A, the calibration plots of peak height $v s$. concentration of saponins $1-4,6,12$ and $8-10$ were found to be linear in the range up to $10 \mu \mathrm{g}$ per injection, and the curves could extrapolated through zero; coefficient of correlation, $r=0.999-0.9999$. The coefficient of variation (c.v.) was found to be $3.97 \%$ for 4 and less than $2.00 \%$ for other saponins.

Saponins 5 and 7 were eluted very fast and could not be separated from each other under condition $\mathrm{A}$, so that quantitative analysis of both saponins was carried out with a different mobile phase: acetonitrile- $0.5 \% \mathrm{H}_{3} \mathrm{PO}_{4}(20: 80)$ [condition B, Fig. 3]. Addition of $\mathrm{H}_{3} \mathrm{PO}_{4}$ was effective for the analysis of both saponins without any perturbation owing to the presence of acidic saponins, especially in the case of continuous analysis of many samples.

Simultaneous analysis of all of the major acidic and neutral saponins including 5 and 7 was achieved by HPLC on an amino column with suppression of ionization by adding $1.0 \%$ $\mathrm{H}_{3} \mathrm{PO}_{4}$ to the mobile phase (condition, C, Fig. 4). Although the separation of 6 from 7,3 from 9 and 2 from 8 was not complete. HPLC under condition $C$ is useful for the qualitative identification of these saponins.

As already mentioned, malony-ginsenosides are rather unstable, being demalonylated on heating. As shown in Fig. 5, heating a solution of the saponin fraction of ginseng in $70 \%$ methanol at $80^{\circ} \mathrm{C}$ for $7 \mathrm{~h}$ resulted in a decrease of malonyl-ginsenosides, 8,9 and 10, and a consequent increase of the corresponding neutral saponins, 1,2 and 3 . The time course of the

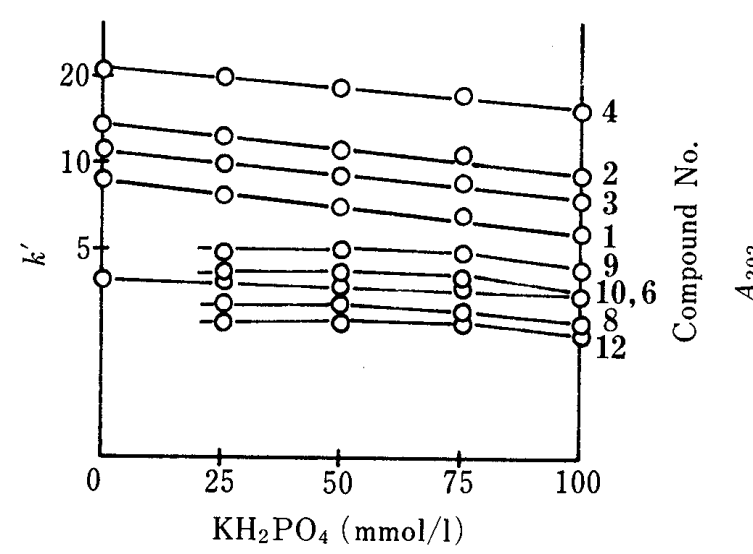

Fig. 1. The Correlation of Concentration of $\mathrm{KH}_{2} \mathrm{PO}_{4}$ with Capacity Ratio $\left(k^{\prime}\right)$ of the Saponins

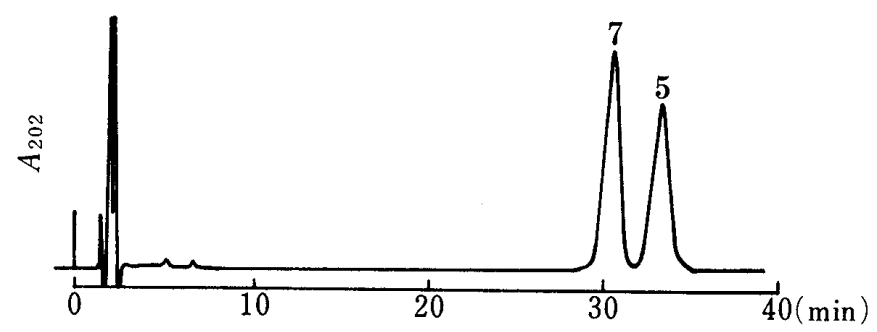

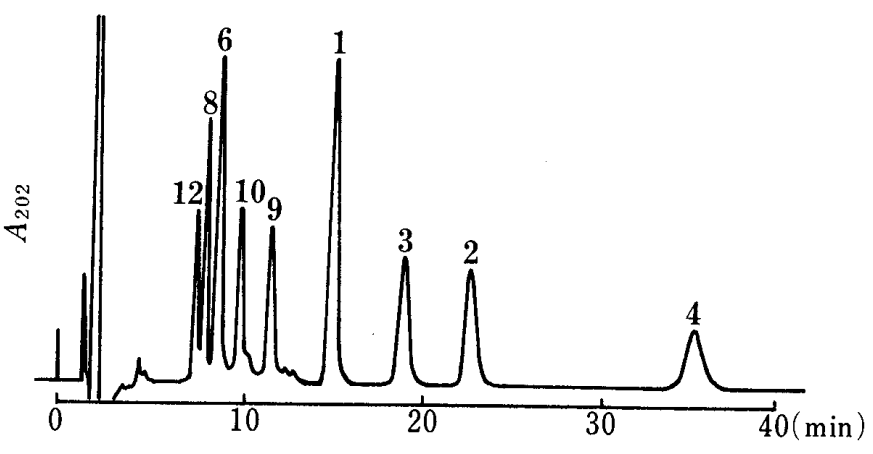

Fig. 2. HPLC Pattern of a Mixture of Saponin Standards (Condition A)

Fig. 3. HPLC Pattern of a Mixture of Saponin Standards (Condition B) 


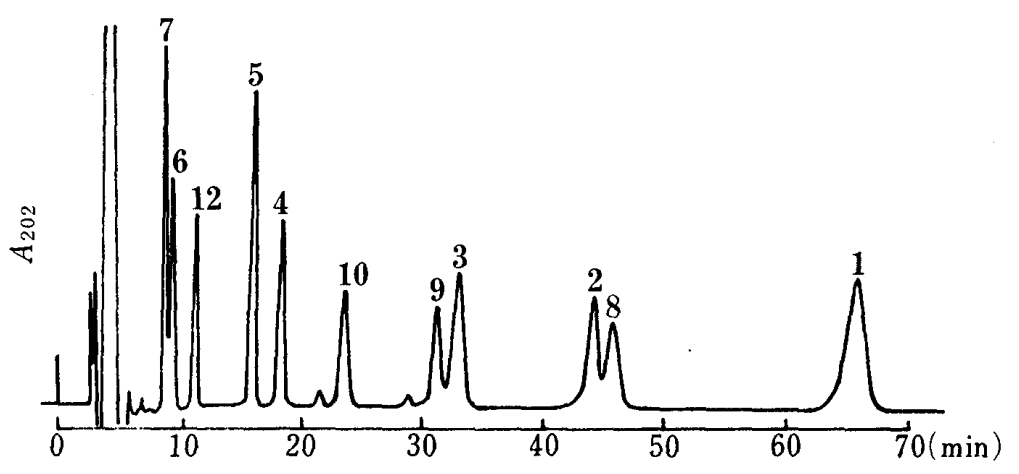

Fig. 4. HPLC Pattern of a Mixture of Saponin Standards (Condition C)

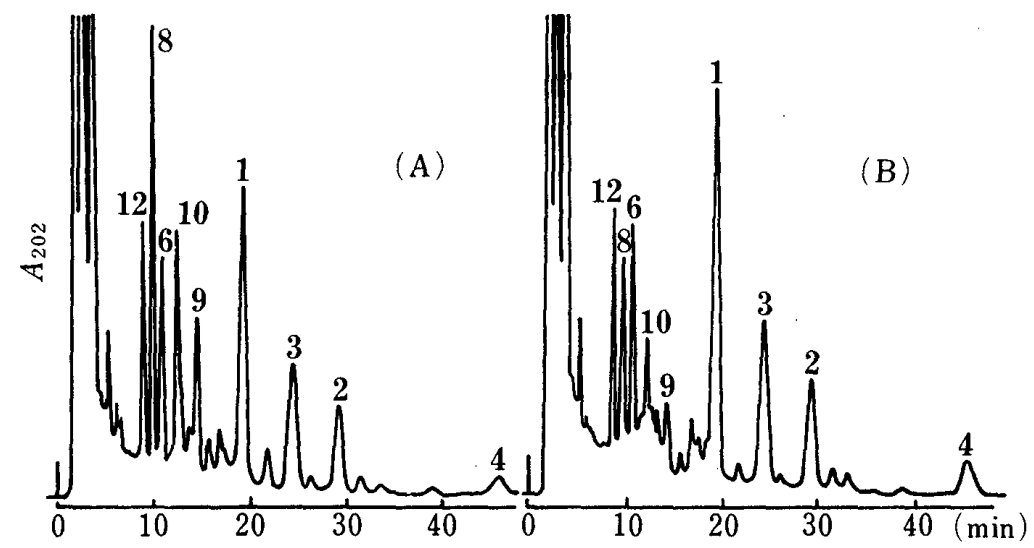

Fig. 5. Thermostability of Malonyl-ginsenosides

HPLC pattern of a solution of a mixture of ginseng saponins. (A) Initial solution in $70 \%$ aqueous $\mathrm{MeOH}$. (B) After heating at $80^{\circ} \mathrm{C}$ for $7 \mathrm{~h}$ (condition A).

Table I. Predicted Residual Ratio (\%) Based on Thermostability of Malonyl-ginsenoside- $\mathrm{Rb}_{1}$ in Solution $(70 \% \mathrm{MeOH})$

\begin{tabular}{cccccc}
\hline & \multicolumn{5}{c}{ Temperature $\left({ }^{\circ} \mathrm{C}\right)$} \\
\cline { 2 - 6 } Time $(\mathrm{h})$ & \multicolumn{6}{c}{3} & 40 & 50 \\
\hline \multirow{2}{*}{40} & 99.99 & 99.72 & 98.74 & 97.43 & 89.93 \\
60 & 99.98 & 99.44 & 97.49 & 94.86 & 79.86 \\
90 & 99.97 & 99.16 & 96.23 & 92.29 & 69.79 \\
120 & 99.97 & 98.88 & 94.97 & 89.72 & 59.73 \\
\hline
\end{tabular}

demalonylation of 8 in $70 \%$ methanol at $4,25,35,40$ and $50{ }^{\circ} \mathrm{C}$ was followed quantitatively by HPLC (condition A, Table I).

Based on these results, for the analysis of the saponins, plant materials were extracted with $70 \%$ methanol at room temperature for $20 \mathrm{~min}$ five times to furnish the complete saponins extract. An aqueous suspension of the extract was chromatographed on MCI gel CHP20P by eluting with water, 30,40 and $50 \%$ aqueous methanol and then with methanol. The HPLC analysis indicated that saponins were not eluted with water, 30 or $40 \%$ methanol but were eluted partly with $50 \%$ methanol and completely with methanol. Consequently, prior to the HPLC analysis, the extract was subjected to chromatography on a small column of MCI gel CHP20P by eluting with water and $40 \%$ methanol successively to remove non- 
saponin substances and then with methanol, affording the saponin fraction.

\section{Comparison of Saponin Contents and Compositions of Several Panax spp.}

By means of this procedure (conditions A and B), the contents of major acidic and neutral saponins in American ginseng ( $P$. quinquefolium L.), Sanchi-ginseng ( $P$. notoginseng (Burk.) F. H. CHEN), Satsuma-ninjin ( $P$. japonicus C.A. MEYER growing in South-Kyushu, Japan) and Panax spp. collected at Central Nepal were determined and compared with those in ginseng. Qualitative identification of each major saponin was confirmed by HPLC (condition C). The minor malonyl-saponin, 11 was only qualitatively identified. The results are listed in Table II.

It is noteworthy that the contents of $\mathbf{8}$ and $\mathbf{1 2}$ in rhizomes of ginseng are high.

American Ginseng - Isolation and identification of saponins from underground parts of this plant were reported by Sanada and Shoji ${ }^{7)}$ and Matsuura et al. ${ }^{8)}$ In the present study, it was found that as in the case of ginseng, ${ }^{9)}$ saponin contents in rhizomes and lateral roots are remarkably higher than those in main roots. Malonyl-ginsenosides $(\mathbf{8}-\mathbf{1 1})$ were detected in main roots as well as lateral roots and rhizomes. The contents of 2, 3, 7, 9 and 10 are lower than those in ginseng and 6 was not detected, while the contents of 1, 4 and 8 in all parts are evidently higher than those in ginseng. The content of $\mathbf{1 2}$ in rhizomes is higher than those of main roots and lateral roots, as in the case of ginseng.

Satsuma-Ninjin $-P$. japonicus is distributed throughout Japan (Japanese name: Chikusetsu-ninjin) and also in China (Chinese name: Zhujie-shen). ${ }^{10)}$

The specimen, named Satsuma-ninjin, growing in South Kyushu, Japan (Kagoshima and Miyazaki prefectures), is not morphologically distinguished from Chikusetsu-ninjin growing in other districts of Japan. However, it has been reported ${ }^{11)}$ that the dammarane saponin composition of Satsuma-ninjin is significantly different from that of Chikusetsu-ninjin, being rather similar to that of Zhujie-shen. None of the major dammarane saponins of ginseng was isolated from Chikusetsu-ninjin, while 1, 3,5 and 7 were isolated from Satsuma-ninjin.

In the present study on Satsuma-ninjin, it was revealed that the lateral roots contain a large amount of the malonyl-saponin (8) and its parent saponin, $\mathbf{1}$, while the contents of $\mathbf{3}$ and 10 in the lateral roots was found to be very low and 2 and 9 were not detected: However, in the

TABle II. Comparison of Saponin Contents $(w / w \%)$ and Composition of Ginseng and Related Plants

\begin{tabular}{|c|c|c|c|c|c|c|c|c|c|c|c|c|}
\hline \multirow[b]{2}{*}{ Saponin } & \multicolumn{3}{|c|}{ Dried ginseng roots } & \multicolumn{3}{|c|}{ American ginseng } & \multicolumn{2}{|c|}{ Satsuma-ninjin } & \multicolumn{3}{|c|}{ Sanchi-ginseng } & \multirow{2}{*}{$\begin{array}{c}\text { Panax spp. } \\
\text { (Central } \\
\text { Nepal) }\end{array}$} \\
\hline & $\begin{array}{c}\text { Rhi- } \\
\text { zomes }\end{array}$ & $\begin{array}{l}\text { Main } \\
\text { roots }\end{array}$ & $\begin{array}{l}\text { Lateral } \\
\text { roots }\end{array}$ & $\begin{array}{c}\text { Rhi- } \\
\text { zomes }\end{array}$ & $\begin{array}{l}\text { Main } \\
\text { roots }\end{array}$ & $\begin{array}{c}\text { Lateral } \\
\text { roots }\end{array}$ & $\begin{array}{c}\text { Rhi- } \\
\text { zomes }\end{array}$ & $\begin{array}{l}\text { Lateral } \\
\text { roots }\end{array}$ & $\begin{array}{c}\text { Rhi- } \\
\text { zomes }\end{array}$ & $\begin{array}{l}\text { Main } \\
\text { roots }\end{array}$ & $\begin{array}{l}\text { Lateral } \\
\text { roots }\end{array}$ & \\
\hline 1 & 0.88 & 0.55 & 2.0 & 1.9 & 1.2 & 2.8 & 1.7 & 4.7 & 4.1 & 3.1 & 1.7 & 2.7 \\
\hline 2 & 0.57 & 0.37 & 1.8 & 0.10 & 0.03 & 0.16 & - & - & 0.17 & 0.03 & - & - \\
\hline 3 & 0.47 & 0.31 & 1.5 & 0.13 & 0.21 & 1.2 & 0.23 & + & - & - & - & - \\
\hline 4 & 0.16 & 0.08 & 0.52 & 0.78 & 0.29 & 1.7 & - & - & 1.3 & 0.84 & 0.40 & 0.60 \\
\hline 5 & 0.57 & 0.35 & 1.4 & 2.0 & 0.64 & 2.2 & 0.04 & 0.70 & 0.50 & 0.21 & 0.27 & 0.02 \\
\hline 6 & 0.15 & 0.11 & 0.17 & - & - & - & - & - & - & - & - & - \\
\hline 7 & 0.38 & 0.45 & 0.25 & 0.20 & 0.09 & 0.17 & 0.09 & 0.27 & 4.7 & 1.6 & 1.8 & 0.09 \\
\hline 8 & 0.69 & 0.41 & 1.2 & 3.0 & 1.1 & 2.9 & + & 2.3 & 0.17 & 0.28 & 0.12 & 1.1 \\
\hline 9 & 0.42 & 0.30 & 1.1 & 0.21 & 0.02 & 0.13 & - & - & - & - & - & - \\
\hline 10 & 0.35 & 0.23 & 0.84 & 0.27 & 0.14 & 0.86 & + & + & - & - & - & - \\
\hline 11 & + & + & + & + & + & + & - & - & - & - & - & - \\
\hline 12 & 1.8 & 0.50 & 0.62 & 0.65 & 0.14 & 0.08 & 2.7 & 1.3 & - & - & - & - \\
\hline 14 & - & - & - & - & - & - & 1.2 & + & - & - & - & - \\
\hline Total $(\%)$ & 6.4 & 3.7 & 11.4 & 9.2 & 3.9 & 12.2 & 6.0 & 9.3 & 10.9 & 6.1 & 4.3 & 4.5 \\
\hline
\end{tabular}


rhizomes, the contents of both $\mathbf{8}$ and $\mathbf{1 0}$ are very low, while that of $\mathbf{3}$ is relatively high. From the rhizomes of Chikusetsu-ninjin, ${ }^{4,12)}$ Zhujie-shen ${ }^{13)}$ and Satsuma-ninjin, ${ }^{11)}$ the glucuronide saponin of 13 named chikusetsusaponin IV (14) has been isolated in a high yield together with a large quantity of 12 . In the present study, only a trace of 14 was detected in lateral roots of Satsuma-ninjin. From the rhizomes, ginsenoside- $\mathrm{Ra}_{3}(\mathbf{1 5}),{ }^{14)}$ the minor dammarane saponin of Ginseng was isolated in a yield of $1.0 \%$.

Sanchi-Ginseng-A number of neutral dammarane saponins have been isolated from this well-known Chinese medicinal plant, while no saponin of 13 has been detected. ${ }^{7{ }^{15)}}$ It is noteworthy that the contents of 1, 4 and 7 are high. In the present study, the presence of a large amount of 8 in the main roots, rhizomes and lateral roots was confirmed, but 3,9 and 10 were not detected.

Panax Spp. Growing in Central Nepal-It has been reported that the saponin composition of rhizomes of the Panax spp. collected near Annapurna, Nepal, the western limit of the distribution of this species, is similar to that of Sanchi-ginseng; no saponin of 13 was detected. ${ }^{16)}$ HPLC analysis of saponins revealed high contents of 1 and its malonylsaponin, 8 in the rhizomes.

\section{Experimental}

Materials - All of the standard saponins in the present study were obtained in our serial studies on Panax spp. The plant materials are as follows. Dried ginseng roots: cultivated in Jilin, China for six years and dried without peeling. American ginseng: cultivated in Nagano prefecture, Japan for three years, and dried without peeling. Satsuma-ninjin: collected in Miyazaki prefecture, Japan, in 1983 and supplied by Mr. Akira Seo and Mr. Shohichiro Seo, Kagoshima, Japan. Sanchi-ginseng: cultivated in Yunnan, China for six years, and dried without peeling. Panax spp. in Central Nepal: collected at Manang (elevation: 3000 m, northern foot of Mt. Annapurna), Nepal, in August 1986 and supplied by Mr. Hideo Fujita, Tokyo, Japan.

Apparatus-A Trirotar-III HPLC apparatus (JASCO, Tokyo, Japan) equipped with Shimadzu SPD-2A variable-wavelength UV detector was used. HPLC conditions were as follows. Condition A: column, Ultron N-C18 (Chromato Packings Center, Kyoto, Japan), particle size, $5 \mu \mathrm{m}$; column size, $4.6 \times 150 \mathrm{~mm}$; mobile phase, $50 \mathrm{~mm}$ $\mathrm{KH}_{2} \mathrm{PO}_{4}$ in $31 \%$ aqueous $\mathrm{CH}_{3} \mathrm{CN}$; flow rate, $1.0 \mathrm{ml} / \mathrm{min}$; detection, $\mathrm{UV} 202 \mathrm{~nm}$; column temperature, $40^{\circ} \mathrm{C}$; chart speed, $2.5 \mathrm{~mm} / \mathrm{min}$. Condition $\mathrm{B}$ : mobile phase, $\mathrm{CH}_{3} \mathrm{CN}-0.5 \% \mathrm{H}_{3} \mathrm{PO}_{4}(20: 80 \mathrm{v} / \mathrm{v})$. Condition C: column, Ultron $\mathrm{NH}_{2}$ (Chromato Packings Center); particle size, $7 \mu \mathrm{m}$; column size, $4.6 \times 250 \mathrm{~mm}$; mobile phase, $\mathrm{CH}_{3} \mathrm{CN}-1.0 \%$ $\mathrm{H}_{3} \mathrm{PO}_{4}(83: 17 \mathrm{v} / \mathrm{v})$. The other conditions were the same as condition A. Acetonitrile (HPLC grade) was purchased from Wako Pure Chem. Ind. (Osaka, Japan). $\mathrm{KH}_{2} \mathrm{PO}_{4}$ and $\mathrm{H}_{3} \mathrm{PO}_{4}$ (guaranteed reagent grade) were purchased from Wako Pure Chem. Ind.

Procedure of Sample Preparation Crushed roots (or rhizomes, $5 \mathrm{~g}$ ) dried in a desiccator containing $\mathrm{P}_{2} \mathrm{O}_{5}$ were extracted 5 times with $30 \mathrm{ml}$ of $70 \% \mathrm{MeOH}$ at room temperature for $20 \mathrm{~min}$ and the combined extracts were concentrated to dryness below $40^{\circ} \mathrm{C}$. An aqueous suspension of the residue was chromatographed on a small column of highly porous polymer (MCI-gel CHP20P, 75-150 $\mu \mathrm{m}, 1 \times 10 \mathrm{~cm}$, Mitsubishi Chemical Industry) by eluting with $\mathrm{H}_{2} \mathrm{O}, 40 \% \mathrm{MeOH}$ and then $\mathrm{MeOH}(100 \mathrm{ml}$ each). The $\mathrm{MeOH}$ eluate was concentrated to dryness and the residue (saponin fraction) was dissolved in $70 \% \mathrm{MeOH}(50 \mathrm{ml})$ for HPLC analysis (sample size; $25 \mu \mathrm{l}$ ).

Isolation of 15 from Rhizomes of Satsuma-Ninjin Rhizomes of Satsuma-ninjin $(3.9 \mathrm{~g})$ were treated as described above. The saponin fraction $(300 \mathrm{mg})$ was dissolved in small amounts of $70 \% \mathrm{MeOH}$ and subjected to preparative HPLC on a hydroxyapatite column (HP40-100, PENTAX, Tokyo, Japan) ${ }^{6}$ by eluting with $77 \% \mathrm{MeCN}$ to give 15 in a yield of $1.0 \%$; this product was identified by comparison of HPLC behavior and nuclear magnetic resonance (NMR) data with those of an authentic sample.

Acknowledgements We are grateful to Mr. M. Takada, Yamajirushi Brew Co., Ltd., Maruko-machi, Nagano, Mr. H. Fujita, Tokyo, and Mr. S. Seo and Mr. A. Seo, Kagoshima, for providing the plant materials.

\section{References}

1) O. Tanaka and R. Kasai, "Progress in the Chemistry of Organic Natural Products," Vol. 46, ed. by W. Herz, H. Grisebach, G. W. Kirby and Ch. Tamm, Springer-Verlag, Vienna, New York, 1984, p. 1; O. Tanaka, R. Kasai and T. Morita, Abstracts of Chinese Medicines, 1, 130 (1986).

2) I. Kitagawa, T. Taniyama, T. Hayashi and M. Yoshikawa, Chem. Pharm. Bull., 31, 3353 (1983). 
3) S. Sanada, N. Kondo, J. Shoji, O. Tanaka and S. Shibata, Chem. Pharm. Bull., 22, 421 (1974).

4) N. Kondo and J. Shoji, Yakugaku Zasshi, 88, 325 (1968); N. Kondo, J. Shoji, N. Nagumo and N. Komatsu, ibid., 89, 846 (1969); N. Kondo, Y. Marumoto and J. Shoji, Chem. Pharm. Bull., 19, 1103 (1971).

5) H. Yamaguchi, H. Matsuura, R. Kasai, K. Mizutani, H. Fujino, K. Ohtani, T. Fuwa and O. Tanaka, Chem. Pharm. Bull., 34, 2859 (1986).

6) R. Kasai, H. Yamaguchi and O. Tanaka, J. Chromatogr., 407, 205 (1987).

7) S. Sanada and J. Shoji, Shoyakugaku Zasshi, 32, 96 (1978).

8) H. Matsuura, R. Kasai, J. X. Wei, J. F. Wang, Y. Saruwatari, T. Fuwa and O. Tanaka, Chem. Pharm. Bull., 30, 4534 (1982).

9) S. Sanada, J. Shoji and S. Shibata, Yakugaku Zasshi, 98, 1048 (1978).

10) H. Hara, Shokubutu Kenkyu Zasshi, 45, 197 (1970).

11) T. Morita, O. Tanaka and H. Kohda, Chem. Pharm. Bull., 33, 3852 (1985).

12) T.-D. Lin, N. Kondo and J. Shoji, Chem. Pharm. Bull., 24, 253 (1976).

13) T. Morita, R. Kasai, O. Tanaka, J. Zhou, T.-R. Yang and J. Shoji, Chem. Pharm. Bull., 30, 4341 (1982).

14) H. Matsuura, R. Kasai, O. Tanaka, Y. Saruwatari, K. Kunihiro and T. Fuwa, Chem. Pharm. Bull., 32, 1188 (1984).

15) M. Z. Wu, Acta Botanica Yunnanica, 1, 119 (1979); J. X. Wei, J. F. Wang, L. Y. Chang and Y. C. Du, Acta Pharmaceutica Sinica, 15, 359 (1980); H. Matsuura, R. Kasai, O. Tanaka, Y. Saruwatari, T. Fuwa and J. Zhou, Chem. Pharm. Bull., 31, 2281 (1983).

16) T. Namba, K. Matsushige, T. Morita and O. Tanaka, Chem. Pharm. Bull., 34, 730 (1986). 